\title{
CARACTERIZACION DE NUEVE GENOTIPOS DE MAIZ (Zea mays L.) EN RELACION A AREA FOLIAR Y COEFICIENTE DE EXTINCION DE LUZ
}

\author{
R.G. CAMACHO'; O. GARRIDO²; M.G. LIMA ${ }^{3}$ \\ 'Departamento de Ciencias de la Tierra y Medioambiente, Universidad Rómulo Gallegos, "El Castrero", San Juan de los \\ Morros, Guárico, Venezuela \\ 2Instituto de Agronomia, Facultad de Agronomia, Universidad Central de Venezuela, "El Limón", Maracay, Venezuela \\ ${ }^{3}$ Departamento de Engenharia Agricola e Solos do CCA/UFPJ, Campus Agricola da Socopo, CEP: 64070-970 - Teresina-PI.
}

RESUMEN: Se caracterizaron nueve materiales genéticos de maíz (Arichuna, Baraure, Braquítico, Experimental-2, Foremaíz PB, FM-6, Obregón, Proseca-71 y Tocorón), en relación con área promedio de hoja por planta, área foliar total por planta (AFT), índice de área foliar (LAF), y coeficiente de extinción de luz (K) a 0,50 m, 1,00 m, 1,50 m, 2,00 m y 2,50 m de altura de la planta(medidos a partir del suelo). Además, estudios de correlación y regresión simple fueron hechos entre rendimiento (R) y IAF. Se encontraron rangos de variación para las diversas variables: Area promedio de hoja por planta (471 $\mathrm{cm}^{2}$ para Foremaíz PB y $606 \mathrm{~cm}^{2}$ para Baraure); AFT $\left(5327 \mathrm{~cm}^{2}\right.$ en Foremaíz PB y $8411 \mathrm{~cm}^{2}$ para Braquítico); IAF (4,26, Foremaiz PB y 6,67, Braquítico); K (0,23 para Braquitico y 0,42, Arichuna); rendimiento (2 $877 \mathrm{~kg} \cdot \mathrm{ha}^{-1}$, Braquítico y $4784 \mathrm{~kg}$.ha-1 en Tocorón). La relación entre rendimiento y IAF no fue significativa( $(r=0,07)$. La asociación de IAF y $K$ fue muy bien descrita por la ley de Beer.

Palabras Claves: maíz, índice de área foliar, coeficiente de extinción de luz, rendimiento.

\section{EVALUATION OF NINE CORN (Zea mays L.) GENOTYPES IN RELATION TO LEAF AREA AND LIGHT EXTINCTION COEFFICIENT}

\begin{abstract}
A field experiment was carried out to evaluate nine corn genotypes (Arichuna, Baraure, Braquitico, Experimental-2, Foremaíz PB, FM-6, Obregón, Proseca-71 and Tocorón) in relation to: mean leaf area per plant, total leaf area per plant (TLA), leaf area inder (LAI), grain yield $(\mathrm{S})$ and light extinction coeficient $(\mathrm{K})$ at $0.50 \mathrm{~m}, 1.00 \mathrm{~m}, 1.50 \mathrm{~m}$, $2.00 \mathrm{~m}$ and $2.50 \mathrm{~m}$ of plant height (from soil to nag leaf). Also, correlation and single regression between $\mathrm{LAI}$ and yield was performed. Significant genotypical differences for all variables were found, except for mean leaf area per plant. Ranging was: mean leaf area per plant (471 $\mathrm{cm}^{2}$ for Foremaíz $P B$ and $606 \mathrm{~cm}^{2}$ for Baraure); TLA $\left(5,327 \mathrm{~cm}^{2}\right.$ for Foremaíz PB and 8, 411 for Braquítico); LAI (4.26, Foremaiz PB and 6.67, Braquítico); K (0.23 for Braquítico and 0.42, Arichuna); Y (2, 877, Braquítico and 4,784 $\mathrm{kg} \cdot \mathrm{ha}^{-1}$ for Tocorón). The relationship between $Y$ and LAI was not significant $(r=0.07)$. The relationship of $L A I$ and $K$ was described very well by Beer's law.
\end{abstract}

Key Words: corn, leaf area index, light extinction coefficient, yield

\section{CARACTERIZAÇĀO DE NOVE GENÓTIPOS DE MILHO (Zea mays L.) EN RELAÇĀO À ÁREA FOLIAR E COEFICIENTE DE EXTINÇÃO DE LUZ}

RESUMO: Foram caracterizados nove materiais genéticos de milho (Arichuna, Baraure, Braquítico, Experimental-2, Foremaiz PB, FM-6, Obregón, Proseca-71 y Tocorón) com relação a área foliar média de folhas por planta(AF), área foliar total por planta (AFT), indice de área foliar (IAF) e coeficiente de extinção de luz (K) a $0,50 \mathrm{~m}, 1,00 \mathrm{~m}, 1,50 \mathrm{~m}, 2,00 \mathrm{~m}$ e 2,50m de altura (a partir do solo). Foram encontrados os seguintes intervalos de variação para as diversas variáveis: área média de folhas por planta (471 $\mathrm{cm}^{2}$ para Foremaíz PB e $606 \mathrm{~cm}^{2}$ Baraure); área foliar total por planta: $5327 \mathrm{~cm}^{2}$ (Foremaíz PB) e $8411 \mathrm{~cm}^{2}$ (Braquítico), indice de área foliar: 4,26 (Foremaiz PB) e 6,67 (Braquítico); coeficiente de extinção de luz: 0,23 (Braquítico) e 0,42 (Arichuna); rendimento: $2877 \mathrm{~kg}^{-h^{-1}}$ (Braquítico) e 4784 kg.ha-1 (Tocorón). Não foi encontrada relação entre o rendimento e o IAF $(r=0,07)$, e a associação do IAF e $K$ foi muito bem explicada pela let de Beer.

Descritores: milho, indice de área follar, coeficiente de extincão de luz, rendimento 5 . 


\section{INTRODUCCION}

El maíz es un alimento de primer orden para un vasto número de personas alrededor del mundo, inclúdos Venezuela y Brasil. La especie está adaptada a áreas de zona tropical, subtropical y templada.

En paises de clima templado se han relatado productividades máximas de $20 t$ de grano por hectárea (FREY, 1971), lo que es debido al mejoramiento genético y al uso de genotipos con altos rendimientos en condiciones donde suelo y agua no constituyen factores limitantes.

El rendimiento del maiz es función de muchos factores medioambientales y de la propia planta, que algunas veces están interrelacionados. Dos de ellos ( la distribución del área foliar y la radiación dentro del dosel de la planta) están relacionados con el proceso fotosintético. Dosel de plantas que reciben la mayor proporción de energia radiante incidente tendrán una eficiencia fotosintética aumentada (WALL \& KANEMASU, 1990).

En el dosel, el índice de área foliar (IAF) es un importante parámetro biofisico para analizar la cantidad de radiación fotosintéticamente absorbida. Un aumento en el IAF proporciona aumento de producción de biomasa; pero, debido al autosombreamiento de las hojas, la tasa fotosintética media por unidad de área foliar decrece (LUCCHESI, 1987).

El objetivo de este trabajo fue estudiar el comportamiento de nueve cultivares de maíz en relación con el IAF y el coeficiente de extinción de luz.

\section{MATERIALES Y METODOS}

Para la realización del trabajo se montó un ensayo en la Estación Experimental Samán Mocho, Facultad de Agronomía, Universidad Central de Venezuela, situada en el Distrito Carlos Arvelo, Estado Carabobo, a $10^{\circ} 15^{\prime} \mathrm{N}$ y $67^{\circ} 39^{\prime} \mathrm{W}$; a $422 \mathrm{~m}$ de altitud; suelos de la serie Valencia, de origen lacustre, ricos en materia orgánica, de textura franco arcillolimosa, estructura granular muy friable, $\mathrm{pH}$ entre 7,1 y 7,5; precipitación anual entre 1100 y $1200 \mathrm{~mm}$ y temperatura media anual del aire $25^{\circ} \mathrm{C}$. Los hibridos crecieron bajo condiciones adecuadas de agua y nutrientes.

Se usaron los siguientes materiales genéticos: 1) Arichuna: (48-S-28 $\times$ Capt. 63-6-1) $\times$ (Eto blanco $2053 \times$ Eto blanco 244); 2) Baraure: (T-11 X
T-12) x (Eto blanco 2053); 3) Braquítico: Compuesto varietal; 4) Experimental 2: Pedigree cerrado; 5) Foremaiz PB: Pedigree cerrado; 6) FM-6: (Lleras III $53 \times 53$ - Sic - 175-2) x (Eto blanco 2053 x Desc. 2 H 29); 7) Obregón: (48-S-38 x 48-S-74) x (Lleras III $53 \times$ Eto blanco 2053); 8) Proseca-71: Pedigree cerrado; 9) Tocorón: Pedigree cerrado.

Se usó un diseño de bloques completos al azar con unidades experimentales de $20 \mathrm{~m}^{2}$ (2 hileras de $10 \mathrm{~m}$ de largo separadas $1 \mathrm{~m}$ entre si). Para las determinaciones de área foliar se procedió de la siguiente manera: se tomaron al azar dos plantas por unidad experimental (una en cada hilera) y se midió largo $y$ ancho de todas las hojas de cada planta; multiplicando largo $x$ ancho $x$ 0,75 (MONTGOMERY, 1911) para determinar área foliar por hoja, lo que permitió obtener el área foliar total por planta y el correspondiente índice de área foliar (dividiendo esta área foliar total entre el área del suelo ocupada por la planta) en plena fase de llenado del grano, sabiendo que la población inicial sembrada fue 80000 plantas por hectárea (área de cada planta $100 \mathrm{~cm} \mathrm{x} \mathrm{12,5}$ $\mathrm{cm})$.

Las mediciones de luz se hicieron utilizando dos sensores: un piranómetro global para la medición de la radiación solar total incidente, localizado a $10 \mathrm{~m}$ fuera de las unidades experimentales, y un radiómetro neto para la determinación de la energía utilizada por el cultivo; esta última medición se hizo en un perfil con dos observaciones en cada una de las siguientes posiciones: desde el suelo hasta 0,50 ; 1,$00 ; 1,50 ; 2,00$ y $2,50 \mathrm{~m}$; determinaciones que se hicieron a los 88 días, después de la siembra, cuando el cultivo estaba en plena fase de llenado de granos. Con la energia medida en microvoltios $(\mu V)$ y conocido el indice de equivalencia del sensor, mediante programa preparado para ser usado em computador, se convertieron los $\mu \mathrm{V}$ en calorías por $\mathrm{cm}^{2}$; através de una curva de calibración, y aplicando la ley de Beer $\left(I=I 0 . e^{\text {teAP }}\right)$ se obtuvieron los valores del coeficiente de extinción (MOTA, 1981), expresados por $K=\left[\ln I_{0}-\ln \Pi\right] / A F$, siendo $I_{0}$ la medida del piranómetro e I las medidas del radiómetro neto, en las respectivas alturas.

Se hicieron análisis de varianza para las siguientes variables: área promedio de hoja por planta, área foliar total por planta(AFT), IAF, rendimiento, valores de $\mathrm{K}$ a: $0,50 \mathrm{~m}-1,00 \mathrm{~m}-1,50 \mathrm{~m}$ $-2,00 \mathrm{~m}$ y $2,50 \mathrm{~m}$ de altura de la planta y análisis de correlación y regresión simple para rendimiento $e$ IAF. 


\section{RESULTADOS Y DISCUSION}

Para la variable área promedio de una hoja (AF) (TABLA 1) no se observaron diferencias significativas entre los materiales genéticos estudiados, a pesar que se observó cierta variabilidad entre ellos.

En cuanto a AFT por planta (TABLA 1) el análisis estadístico reveló diferencias entre los materiales genéticos, existiendo un grupo integrado por Braquítico, Proseca-71, Baraure, Tocorón y FM6, materiales que producen gran cantidad de AFT por planta; de ellos, el Braquítico es un material de porte enano, que produce gran número de hojas y muy anchas. El rango de variación fue de 5327 (Foremaíz PB) a $8411 \mathrm{~cm}^{2}$ (Braquítico). DURÃES (1993) reportó valores de 5441 a 6647 y $6739 \mathrm{~cm}^{2}$ de área foliar por planta en tres cultivares de maíz, a 91 días después de la siembra y con una densidade de 77700 plantas.ha ${ }^{-1}$.

El estudio del LAF mostró diferencias sig. nificativas entre los materiales genéticos bajo estudio (TABLA 1). Existe un grupo que produjo valores de IAF muy altos, integrado por los mismos materiales que presentaron los mayores valores de AFT y, ahora, se agrega el híbrido Obregón. Es razonable que los materiales genéticos se comporten en forma similar para estas dos variables, ya que el IAF es una consecuencia directa del área foliar total por planta, resultados que concuerdan con aquellos obtenidos por DURÃES (1993).

En este trabajo el IAF varió entre 4,26 (Foremaíz-PB) a 6,67 (Braquítico). Estudios conducidos por LEAL (1993) demostraron que el mayor índice de área foliar $(\mathrm{IAF}=6,70)$ fue observado em parcelas con 80000 plantas de maiz por hectárea.

Variación genotípica fue encontrada para el rendimiento de grano (TABLA 1). Como puede notarse, existe un grupo superior integrado por Tocorón, Obregón y FM-6, con rendimientos superiores a 4,6 tha $a^{-1}$, y éstos son tres de los seis hibridos que producen los mayores valores de $\operatorname{IAF}(5,99$; 5,34 y 5,80 , respectivamente). Existe un grupo con rendimientos intermedios $\left(4,1\right.$ a 4,4 tha $\left.^{-1}\right)$, integrado por los híbridos Baraure, Arichuna y Proseca-71 (que presentaron valores de IAF de 5,90, 5,14 y 6,46, respectivamente). Foremaiz-PB ( $I A F=4,26)$ produce 3,9 t.ha $^{-1}$, Experimental-2 $(\mathrm{IAF}=4,50)$ rinde 3,1 tha ', y finalmente Braquítico (IAF $=6,67$ ) produce 2,9 t.ha'.

El análisis de correlación sugiere la existencia de una relación funcional poco estrecha entre rendimiento y $\operatorname{LAF}(r=0,07)$, contrario a lo señalado por algunos autores (EIK \& HANWAY,
1966, NUÑEZ \& KAMPRATH, 1969; SCARSBROOK \& DOSS, 1973; WALL \& KANEMASU, 1990; DURÃES, 1993).

Em maiz, NUÑEZ \& KAMPRATH (1969) obtuvieron máximo rendimiento con un IAF de 3,5, permaneciendo constante hasta un IAF de 4,5 cuando la suplencia de $\mathrm{N}$ es adecuada. En contraposición, YOSHIDA (1972) mostró que un IAF de 5 es óptimo para el cultivo de maíz. En este trabajo los valores de IAF variaron entre 4,26 (Foremaiz-PB) e 6,67 (Braquítico), mostrando que los cultivares con mayores rendimientos (FM-6, Obregón y Tocorón) tienen, también, IAF elevados.

Es posible que los bajos rendimientos en grano observados en la presente investigación sean ocasionados por utilizar una población de 80000 plantas por hectárea, ya que ha sido indicado que valores de IAF próximos de 3,5 tienen una relación lineal con el rendimiento (EIK \& HANWAY, 1966; NUÑES \& KAMPRATH, 1969); no obstante, SCARSBROOK \& DOSS (1973) encontraron aumentos del rendimiento en grano con incremento de la población hasta 80000 plantas por hectárea. A medida que el IAF aumenta, las hojas inferiores son más sombreadas y, consecuentemente, la tasa fotosintética media de todo el área foliar es disminuida (LUCCHESI, 1987). NUÑEZ \& KAMPRATH (1969) han sugerido que el rendimiento en grano es dependiente del AFT, y la disminución del área foliar (AF) com el aumento de la población de plantas se debe a la existencia de competencia por luz $\mathrm{y} / \mathrm{o} \mathrm{CO}$.

En la TABLA 2 se presentan los valores del coeficiente de extinción de luz $(K)$ en el perfil estudiado.

Los análisis de varianza revelaron diferencias significativas entre los distintos materiales genéticos a $0,50 \mathrm{~m}, 1,50 \mathrm{~m}$ y $2,00 \mathrm{~m}$ de altura de las plantas (a partir de la superficie del suelo); no obstante, para todos ellos hay una misma tendencia a producir valores de $\mathrm{K}$ que son bajos en la parte superior pero que se van incrementando a medida que se desciende en el perfil, lo que es razonable si se toma en cuenta que $\mathrm{K}$ mide la luz que atraviesa el dosel. Estos resultados concuerdan con aquellos observados por SCARSBROOK \& DOSS (1973).

Analizando lo que acontece en cada estrato, se puede observar que, a 2,50 $\mathrm{m}$ los valores de $\mathrm{K}$ son bajos, hay muy pocas hojas, por lo que la luz penetra fácilmente el dosel; aquí el comportamiento de los materiales genéticos es semejante, no hubo significación estadística para la variabilidad que se observó. 
TABLA 1 - Promedios de área foliar (AF), área foliar total por planta (AFT), indice de área foliar (IAF) y rendimiento $(R)$, de nueve genotipos de maiz.

\begin{tabular}{|c|c|c|c|c|}
\hline Genotipos & AF & AFT & IAF & $\mathrm{R}$ \\
\hline \multicolumn{4}{|c|}{$\mathrm{cm}^{2}$} & $\mathrm{~kg} \cdot \mathrm{ha} \mathrm{a}^{-1}$ \\
\hline Arichuna & $521 \mathrm{a}$ & $6428 b$ & 5,14 a & $4248 b$ \\
\hline Baraure & $606 a$ & $7383 \mathrm{ab}$ & 5,90 a & $4409 \mathrm{~b}$ \\
\hline Braquítico & $596 \mathrm{a}$ & 8411 a & $6,67 \mathrm{a}$ & $2877 d$ \\
\hline Experimental-2 & $518 \mathrm{a}$ & $5626 b$ & $4,50 \mathrm{~b}$ & $3118 \mathrm{c}$ \\
\hline Foremaíz-PB & $471 \mathrm{a}$ & $5327 b$ & $4,26 \mathrm{~b}$ & $3940 \mathrm{c}$ \\
\hline FM-6 & $601 a$ & $7258 \mathrm{ab}$ & $5,80 \mathrm{a}$ & $4626 \mathrm{a}$ \\
\hline Obregón & $558 \mathrm{a}$ & $6671 b$ & $5,34 \mathrm{a}$ & $4646 a$ \\
\hline Proseca-71 & $595 a$ & $8059 a$ & 6,46 a & $4132 \mathrm{~b}$ \\
\hline Tocorón & $571 \mathrm{a}$ & $7496 \mathrm{ab}$ & 5,99 a & $4786 \mathrm{a}$ \\
\hline $\mathrm{dms}$ & - & 1651 & 1,46 & 279 \\
\hline
\end{tabular}

Valores dentro de cada columna seguidos por la misma letra no difieren significativamente al nivel 0,05 de acuerdo con la prueba de Tukey.

$\mathrm{dms}=$ diferencia mínima significativa.

TABLA 2 - Coeficientes de extinción de luz $(\mathrm{K})$ y valores promedios $(\overline{\mathrm{X}})$ en el dosel de nueve genotipos de maiz.

\begin{tabular}{lllllll}
\hline \hline Genotipos & $0,50 \mathrm{~m}$ & $1,00 \mathrm{~m}$ & $1,50 \mathrm{~m}$ & $2,00 \mathrm{~m}$ & $2,50 \mathrm{~m}$ & $\overline{\mathrm{X}}$ \\
\hline Arichuna & $0,53 \mathrm{a}$ & $0,50 \mathrm{a}$ & $0,47 \mathrm{a}$ & $0,41 \mathrm{a}$ & $0,21 \mathrm{a}$ & 0,42 \\
Baraure & $0,44 \mathrm{~b}$ & $0,41 \mathrm{a}$ & $0,38 \mathrm{a}$ & $0,32 \mathrm{a}$ & $0,20 \mathrm{a}$ & 0,35 \\
Braquítico & $0,34 \mathrm{~b}$ & $0,29 \mathrm{a}$ & $0,23 \mathrm{~b}$ & $0,17 \mathrm{~b}$ & $0,13 \mathrm{a}$ & 0,23 \\
Experimental-2 & $0,48 \mathrm{~b}$ & $0,44 \mathrm{a}$ & $0,33 \mathrm{a}$ & $0,26 \mathrm{a}$ & $0,21 \mathrm{a}$ & 0,34 \\
Foremaíz-PB & $0,46 \mathrm{~b}$ & $0,44 \mathrm{a}$ & $0,32 \mathrm{a}$ & $0,27 \mathrm{a}$ & $0,18 \mathrm{a}$ & 0,33 \\
FM-6 & $0,52 \mathrm{a}$ & $0,50 \mathrm{a}$ & $0,47 \mathrm{a}$ & $0,37 \mathrm{a}$ & $0,19 \mathrm{a}$ & 0,41 \\
Obregón & $0,50 \mathrm{a}$ & $0,46 \mathrm{a}$ & $0,43 \mathrm{a}$ & $0,35 \mathrm{a}$ & $0,20 \mathrm{a}$ & 0,39 \\
Proseca-71 & $0,54 \mathrm{a}$ & $0,51 \mathrm{a}$ & $0,29 \mathrm{~b}$ & $0,24 \mathrm{~b}$ & $0,17 \mathrm{a}$ & 0,35 \\
Tocorón & $0,57 \mathrm{a}$ & $0,52 \mathrm{a}$ & $0,38 \mathrm{a}$ & $0,26 \mathrm{a}$ & $0,17 \mathrm{a}$ & 0,38 \\
\hline dms & 0,08 & & 0,16 & 0,12 & - & \\
\hline \hline
\end{tabular}

Valores dentro de cada columna seguidos por la misma letra no difieren significativamente al nivel 0,05 de acuerdo con la prueba de Tukey.

$\mathrm{dms}=$ diferencia mínima significativa.

A $2,00 \mathrm{~m}$ los valores de $\mathrm{K}$ son más altos, lo que indica que pasa menos luz; la gran variabilidade que reveló el análisis estadístico la proporcionaron Braquítico $(\mathrm{K}=0,17)$ y Proseca-71 $(\mathrm{K}=0,24)$. Esto se debe a que en el estrato entre 2,00 y $2,50 \mathrm{~m}$ existen muy pocas hojas en Proseca71 y ninguna en Braquítico $(1,80 \mathrm{~m}$ de altura promedio). A $1,50 \mathrm{~m}$ siguen creciendo los valores de $K$, lo cual es esperable, y de nuevo la significación estadística la introducen Braquítico y Proseca-71 ( menor proporción de hojas de estos materiales en este estrato). A 1,00 m no se encontraron diferencias estadísticas para la variabilidad del material genético, sin embargo el Braquítico sigue proporcionando el valor de K más bajo, no obstante el de Proseca71 es uno de los más altos.

A $0,50 \mathrm{~m}$, todos los materiales genéticos producen valores de $\mathrm{K}$ superiores a 0,50 , sólo están por debajo de esta cifra Experimental-2 $(0,48)$, Foremaiz-PB $(0,46)$, ambos com IAF de los más bajos $(4,26$ y 4,50 , respectivamente), Baraure $(0,44)$ que a pesar de producir uno de los mayores valores de 
IAF $(5,9)$, su número de hojas promedio por planta es de los más bajos, es decir, tiene una buena distribución de hojas con entrenudos largos (datos no mostrados) $y$, finalmente, Braquítico $(0,34)$, que es un material de porte enano, con elevado índice de área foliar (IAF = 6,67). Proseca-71, que en los estratos superiores produjo valores de $\mathrm{K}$ muy pequeños, en estos dos últimos estratos produce valores altos, debido a que concentra en estos estratos más bajos $(0,50 \mathrm{~m}$ a $1,00 \mathrm{~m}$ y $1,00 \mathrm{~m}$ a $1,50 \mathrm{~m})$ mayor cantidad de hojas.

Ha sido propuesto un $\mathrm{K}$ de 0,3 a 0,5 para una comunidad herbácea de hojas erectas y de 0,7 a 1,0 para hojas horizontales (SAEKI, 1960). En este trabajo los valores de $K$ variaron entre 0,23 (Braquítico) y 0,42 (Arichuna), lo que parece indicar que todos los cultivares son de hojas erectas.

Todo este análisis lleva a inferir que el problema del área foliar y su influencia sobre la producción de granos, está grandemente influenciado más que por la cantidad de área foliar como tal, más bien por la forma como se distribuye este área foliar a lo largo del tallo (ALLEN et al., 1964), y de la disposición de las hojas en el tallo (LOOMIS \& WILLIAMS, 1969).

\section{CONCLUSIONES}

1) No fue encontrada relación significativa entre IAF y $\mathrm{R}$, debido a la competencia por luz $\mathrm{y} / \mathrm{o} \mathrm{CO}_{2}$ en las hojas inferiores;

2) Los híbridos FM-6, Obregón y Tocorón se destacaron por presentar valores elevados para las variables IAF, $K$ y $R$, lo que indica mayor eficiencia de conversión de energía en fotoasimilados, cuando son usadas altas densidades de población;

3) La técnica que relaciona IAF y $\mathrm{K}$, utilizada para calcular la distribución espacial de la luz dentro de la comunidad de plantas de maiz, fue muy bien descrita por la ley de Beer.

\section{REFERENCIAS BIBLIOGRAFICAS}

ALLEN, L.H.; YOCUM, C.S.; LEMON, E.R. Photosynthesis under field conditions. VII. Radiant energy exchanges within a corn canopy and implications in water use efficency. Agronomy Journal, Madison, v.56, p. 253$259,1964$.

DURÃES, F.O.M. Fatores ecofisiológicos afetando o comportamento de milho em semeadura tardia (safrinha) no Brasil Central. Piracicaba, 1993. 91p. Tese (Doutorado) - Escola Superior de Agricultura "Luiz de Queiroz", Universidade de Sa Paulo.

Sci. agric., Piracicaba, 52(2):294-298, mai./ago. 1995
EIK, K.; HANWAY, J.J. Leaf area in relation to yield of com grain. Agronomy Joumal, Madison, v.58, p. 16-18, 1966.

FREY, K.J. Improving crop yields through plant breeding. In: AMERICAN SOCIETY OF AGRONOMY. Moving of the yield plateau, Madison, 1971. p.175-193. (ASA. Special Publication, 20).

LEAL, B. G. Caracterizaçăo da radiaçăo, análise de crescimento e do desenvolvimento de milho (Zea mays L.) em três densidades de plantio. Viçosa, 1993. 55p. Dissertação (Mestrado) - Universidade Federal de Viçosa.

LUCCHESI,A.A. Fatores da produção vegetal. In: CASTRO, P.R.C.; FERREIRA, S.O.; YAMADA, T. (Ed.). Ecofisiologia da produçzo agricola. Piracicaba: Associaçăo Brasileira para Pesquisa da Potassa e do Fosfato, 1987, p.1-11.

LOOMIS, R.S.; WILLIAMS, W.A. Productivity and the morphology of crop stand: patterns with leaves. In: EASTIN, J.D. (Ed.) Physiological aspects of crop yield. Madison: ASA, 1969. p.27-47.

MONTGOMERY, E.G. Correlation studies of com. Nebraska Agricultural Station Annual Report, Lincoln, v. 24, p.108-159, 1911.

MOTA, F. S. Meteorologia agricola. São Paulo, Nobel, 1981. $376 \mathrm{p}$.

NUÑES, R. \& KAMPRATH, E. Relationships between N response, plant population and row width on growth and yield of corn. Agronomy Journal, Madison, v. 61, p. 279 $282,1969$.

SAEKI, T. Interrelationships between leaf amount, light distribution, and total photosynthesis in a plant community. Botanical Magarine, Tokyo, v. 57, p. 593-596, 1960.

SCARSBROOK, C.E.; DOSS, B.D. Leaf area and radiation as related to corn yield. Agronomy Journal, Madison, $v$. 65, p. 459-461, 1973.

WALL, G.W.; KANEMASU, E.T. Carbon dioxide exchange rates in wheat canopies. II. Photosynthetic and phytomass production efficiencies. Agricultural and Forest Meteorology, Amsterdam, v. 49, p.103-122, 1990.

YOSHIDA, S. Physiological aspects of grain yield. Annual Review of Plant Physiolozy, Palo Alto, v.23, p. 437-464, 1972.

Recebido para publicação em 18.07.95

Aceito para publicação em 28.08.95 